\title{
Disruption of CTCF at the miR-125b1 locus in gynecological cancers
}

Ernesto Soto-Reyes ${ }^{1}$, Rodrigo González-Barrios' ${ }^{1}$, Fernanda Cisneros-Soberanis ${ }^{1}$, Roberto Herrera-Goepfert ${ }^{2}$, Víctor Pérez ${ }^{2}$, David Cantú ${ }^{1}$, Diddier Prada ${ }^{1}$, Clementina Castro ${ }^{1}$, Félix Recillas-Targa ${ }^{3}$ and Luis A Herrera ${ }^{1,4^{*}}$

\begin{abstract}
Background: In cancer cells, transcriptional gene silencing has been associated with genetic and epigenetic defects. The disruption of DNA methylation patterns and covalent histone marks has been associated with cancer development. Until recently, microRNA (miRNA) gene silencing was not well understood. In particular, miR-125b1 has been suggested to be an miRNA with tumor suppressor activity, and it has been shown to be deregulated in various human cancers. In the present study, we evaluated the DNA methylation at the CpG island proximal to the transcription start site of miR-125b1 in cancer cell lines as well as in normal tissues and gynecological tumor samples. In addition, we analyzed the association of CTCF and covalent histone modifications at the miR-12561 locus.
\end{abstract}

Methods: To assess the DNA methylation status of the miR-125b1, genomic DNA was transformed with sodium bisulfite, and then PCR-amplified with modified primers and sequenced. The miR-125b1 gene expression was analyzed by qRT-PCR using U6 as a control for constitutive gene expression. CTCF repressive histone marks abundance was evaluated by chromatin immunoprecipitation assays.

Results: The disruption of CTCF in breast cancer cells correlated with the incorporation of repressive histone marks such H3K9me3 and H3K27me3 as well as with aberrant DNA methylation patterns. To determine the effect of DNA methylation at the CpG island of miR-125b1 on the expression of this gene, we performed a qRT-PCR assay. We observed a significant reduction on the expression of miR-125b1 in cancer cells in comparison with controls, suggesting that DNA methylation at the CpG island might reduce miR-125b1 expression. These effects were observed in other gynecological cancers, including ovarian and cervical tumors.

Conclusions: A reduction of miR-125b1 expression in cancers, correlated with methylation, repressive histone marks and loss of CTCF binding at the promoter region.

Keywords: CTCF, miR-125b1, Epigenetic, Cancer, Promoter, MicroRNA, Breast cancer

\section{Background}

MicroRNAs (miRNAs) are a broad family of small noncoding RNAs and are involved in multiple cellular processes [1]. In mammals, miRNAs have been predicted to control the transcriptional activity of more than $60 \%$ of protein-encoding genes [2]. Therefore, miRNAs represent a new component associated with the regulation of gene expression [3]. The study by Sato and collaborators

\footnotetext{
* Correspondence: herreram@biomedicas.unam.mx

'Unidad de Investigación Biomédica en Cáncer, Instituto Nacional de Cancerología (INCan)-Instituto de Investigaciones Biomédicas, Universidad Nacional Autónoma de México (UNAM), México, DF, México Full list of author information is available at the end of the article
}

has gained importance because many miRNAs function as tumor suppressor genes and oncogenes and because their deregulation can lead to the development of cancer [3]. This phenomenon has been observed in lung, colon and breast cancer, among others. The deregulation of miRNAs is associated with both genetic and epigenetic defects $[4,5]$. Some miRNAs have been described to have an oncogenic function, such as $m i R-17-92$, $m i R$ 155 and $m i R-372-373$, and can influence cell proliferation, whereas others present tumor suppressor gene activity, e.g., as $m i R-34, m i R-26 a$ and $m i R-125 b$ [2,6-8].

In particular, the expression of $m i R-125 b 1$ was observed to be decreased in glioblastoma, prostate

\section{Biomed Central}


cancer, ovarian cancer and breast cancer. Interestingly, in vitro studies suggest that miR-125b1 targets HER2/ neu and ESR1 genes, two genes important for the diagnosis and treatment of breast cancer $[9,10]$. One of the mechanisms associated with epigenetic silencing of miR125b1 is DNA methylation [8]. Particularly, a hypermethylation of a CpG island located in proximity to the transcription initiation site was observed in cell lines and in tissue samples from patients with breast cancer $[8,11]$.

It is now known that a multifunctional CCCTC-binding factor (CTCF) can serve as a barrier against the spread of DNA methylation and histone repressive marks over promoter regions of tumor suppressor genes. CTCF has been involved in many aspects of epigenetic regulation, such as $\mathrm{X}$ chromosome inactivation, genomic imprinting, regulation of non-coding transcripts and the structure and function of repeated elements $[12,13]$.

CTCF has been involved in the epigenetic regulation of genes related to cell cycle control, such as BRCA1, $\mathrm{Rb}, \mathrm{p} 16$ and p53 [14-18]. Recently, CTCF has also been shown to be involved in the regulation of miRNAs [19]. Therefore, CTCF may be able to protect against DNA methylation and the covalent incorporation of negative marks on histones in miRNAs with CpG islands [19].

The aim of the present study was to evaluate the DNA methylation at the CpG island proximal to the transcription start site of $m i R-125 b 1$ in cancer cell lines as well as in normal tissues and gynecological tumor samples. In addition we analyzed the association of CTCF and covalent histone modifications at the miR-125b1 locus.

We found that the miR-125b1 CpG island is methylated in cancer cells. The disruption of CTCF combined with DNA methylation and the gain of repressive histone covalent marks, such as H3K9me3 and H3K27me3, result in the miRNA gene silencing.

\section{Methods}

\section{Cell culture conditions}

MCF-7, SK-BR-3, MCF10A and MDA-MB-231 cells were cultured in Dulbecco's modified Eagle's medium DMEM (Invitrogen) with 10\% fetal bovine serum (Invitrogen) and 1\% penicillin/streptomycin (Invitrogen, Carlsbad, CA, USA). All cells were grown in a humidified incubator at $37^{\circ} \mathrm{C}$ with $5 \% \mathrm{CO}_{2}$.

\section{Tissue specimens and processing}

All breast, ovarian and cervical cancer samples were obtained from the Instituto Nacional de Cancerología de México and classified according to the American Joint Committee on Cancer (AJCC) using the tumor-lymph node-metastasis (TNM) system. Diagnosis was given by trained pathologists from the institute based on WHO classification. Nine breast cancer samples, four ovarian cancer samples and three cervical cancer samples were obtained prospectively. These samples were obtained from untreated patients. As controls we analyzed three normal breast samples from mammoplasty surgeries, one normal ovarian sample from a hysterectomy indicated by cystocele, and one normal cervical sample from a healthy woman. Fresh samples were preserved at $-20^{\circ}$ $\mathrm{C}$ in RNAlater (Qiagen). Tissue sections were stained with $H \& E$ and were used only if they contained more than $70 \%$ cancer cells. This study was approved by the ethical committee of the Instituto Nacional de Cancerología (approval numbers 011/034/IBI and CB/727).

\section{Bisulfite sequencing and MS-PCR for the miR-125b1 promoter}

DNA from tumor samples and cell lines were obtained by phenol/chloroform extraction. Two micrograms of genomic DNA was modified using the EZ methylation kit (ZYMO). For sequencing, modified DNA was subjected to PCR using oligonucleotides to amplify from the 573 to 303 positions upstream the transcription start site of miR-125b1 (Entrez Gene: 406911). We used the following primers for amplification (270 bp), F: 5'TGGTGTTATAGGAGGTTGTG-3'; and R: 5'-ACCCAAATTTTTAAAACCATAA-3'. PCR products were purified with the DNA clean and concentrator kit (ZYMO Research). The products were cloned into the pGEM-T-Easy vector (Promega). At least sixth clones were selected randomly for DNA sequencing.

MS-PCR was performed with DNA treated with sodium bisulfite. The following primers were designed using MethPrimer software. For methylation analysis MSPmet125b1F 5'-TGGTGTATCGTTTTTTGTTTTC3' and MSPmet125b1R 5'-ACCCATTCGAAACGAAAC, and for unmethylated analysis MSPunm125b1F 5'ATTTGGTGTATTGTTTTTTGTTTTT and MSPunm125b1R 5'-CTCACCCATTCAAAACAAAAC. As a positive control, $1 \mu \mathrm{g}$ of DNA from lymphocytes of a healthy donor was methylated in vitro (IVD) for eight hours using SssI methyltransferase (NEB, Beverly, MA).

\section{ChIP assay}

Standard chromatin immunoprecipitation (ChIP) assays were performed as previously reported [18] with $4 \mu \mathrm{g}$ of the following antibodies: H3K4me2 (07-030, Millipore); H3K9me2 (ab8898, Abcam); H3K27me3 (ab6002, Abcam), and CTCF (07-729, Millipore). As negative control we employed a normal rabbit IgG (sc-2027, Santa Cruz Biotechnology). Immunoprecipitated DNA was analyzed by PCR using specific primers to analyze the four genomic regions, including the miR-125b1 promoter. The 5' p53 gene promoter was used as positive control for CTCT's ChIP [18]; and for the H3K27me3 histone mark we analyzed the Myelin transcription 
factor 1 distal promoter (MYT1) [20]. Primer sequences used for ChIP assays are available by request.

\section{Quantitative real-time RT-PCR (qRT-PCR)}

Total RNA was extracted with Trizol (Invitrogen). The miRNA levels were determined by qRT-PCR performed with cDNA generated from $10 \mathrm{ng}$ of total RNA using the $\operatorname{TaqMan}^{\circledR}$ miRNA Reverse Transcription Kit (Applied Biosystems). To evaluate the expression of the mature miR-125b1, we employed the TaqMan ${ }^{\circledR}$ primer ID 002378 (Applied Biosystems). The relative amount of miR-125b1 was normalized against U6 snRNA (ID 001093), and the fold change was calculated by the 2$\Delta \Delta \mathrm{Ct}$ method. The amplification and detection of specific products were performed with the ABI PRISM 7000 system (Applied Biosystems).

\section{Statistical analysis for qRT-PCR}

Data from at least four independent experiments are expressed as the mean \pm standard deviation. The differences between groups were analyzed using the Student's $t$-test comparing the cancer cell lines with a nontumorigenic epithelial cell line (MCF10A), and breast tumor with normal breast samples. Data were considered significant if $p<0.001$.

\section{Results}

DNA methylation profile of the miR-125b1 CpG island in breast cancer cell lines

As a first approach, we conducted an in silico study of the locus of $m i R-125 b 1$. This miRNA is located on the long arm of chromosome 11 (11q24.1). Let-7a2 miRNAs and $m i R-100$ are located upstream of this gene. In addition, there is a CpG island upstream from the transcription start site of $m i R-125 b 1$ (Figure 1A). The DNA methylation status of the $\mathrm{CpG}$ island was assessed through sodium bisulfite conversion and MS-PCR in various transformed cell lines (Figure 1B). Using synthetic oligonucleotides modified for DNA methylation analysis, we determined that the CpG island associated with $m i R-125 b 1$ is methylated in most breast tumor cell lines, with the exception of SK-BR-3 (Figure 1B, C). DNA from the non-transformed breast cell line MCF10A and DNA methylated in vitro by the enzyme Sss I (IVD) were used as controls (Figure 1B). These data suggest that DNA methylation of the $m i R-125 b 1$ CpG island primarily occurs in transformed cell lines (i. e., MDA-MB-231 and MCF7). To confirm the DNA methylation status, we cloned and sequenced the product of sodium bisulfite conversion (Bs-seq) for the MDA-MB-231, MCF7, SK-BR-3 and MCF10A cell lines (Figure 1C). The results showed that these cell lines had different densities of DNA methylation, with partial methylation in MDA-MB-231 and in MCF7. In contrast,
SK-BR-3 and the control, MCF10A, showed no methylation at the $m i R-125 b 1$ promoter. These results suggest that DNA methylation of the $m i R-125 b 1 \mathrm{CpG}$ island occurs mostly in transformed cells. In order to determine the effect of DNA methylation at the CpG island of $m i R-125 b 1$ on the expression of this gene, we performed a qRT-PCR assay (Figure 1D). We observed a significant reduction on the expression of $m i R-125 b 1$ in MCF7 and MDA-MB-231 in comparison with MCF10A, suggesting that DNA methylation at the $\mathrm{CpG}$ island might reduce $m i R-125 b 1$ expression (Figure 1D). To confirm this hypothesis, we extended our study to samples from human gynecological tumors.

\section{DNA methylation profile at the miR-125b1 CpG island in primary gynecological tumor samples}

Tumor samples were collected from breast $(n=9)$, ovarian $(n=4)$ and cervical tumors $(n=3)$ DNA was extracted from these samples and treated with sodium bisulfite. Subsequently, the DNA methylation status of the promoter of $m i R-125 b 1$ was analyzed by MS-PCR. The methylation status for the breast tumor samples varied, $23 \%$ were totally methylated, $66 \%$ were partially methylated and only $11 \%$ showed no DNA methylation at the $\mathrm{CpG}$ island region of $m i R-125 b 1$. As a control, we used normal breast tissue samples obtained from aesthetic mammoplasties. These control samples were analyzed by MS-PCR and Bs-seq, and no DNA methylation was detected in them (Figure 2A, B).

All together, these results suggest that DNA methylation at the CpG island of $m i R-125 b 1$ occurs in tumors and could be involved in cancer development.

We then used this assay to analyze the methylation status in four ovarian cancer samples and three from cervical cancer. We observed DNA methylation of miR$125 b 1$ in all samples from gynecological tumors except for one breast cancer sample, suggesting that this epigenetic modification is present only in transformed cells. Therefore, we evaluated the effect of the methylation at the CpG island on the expression of $m i R-125 b 1$ in breast cancer samples by qRT-PCR. We found a significant decrease in miR-125b1 expression in breast cancer samples, $\mathrm{BC} 1$ and $\mathrm{BC} 8$, and a marginal reduction in BC9 sample compared with normal breast tissue. These results suggest that DNA methylation at the $m i R-125 b 1$ CpG island decreases significantly the miRNA in human breast cancer samples. While the marginal reduction observed in sample BC9 could suggest that other epigenetic process might also be involved.

\section{Characterization of CTCF and histone marks across the miR-125b1 locus in normal and breast tumor samples}

Because CTCF has been proposed to protect genomic regions against DNA methylation, we decided to 


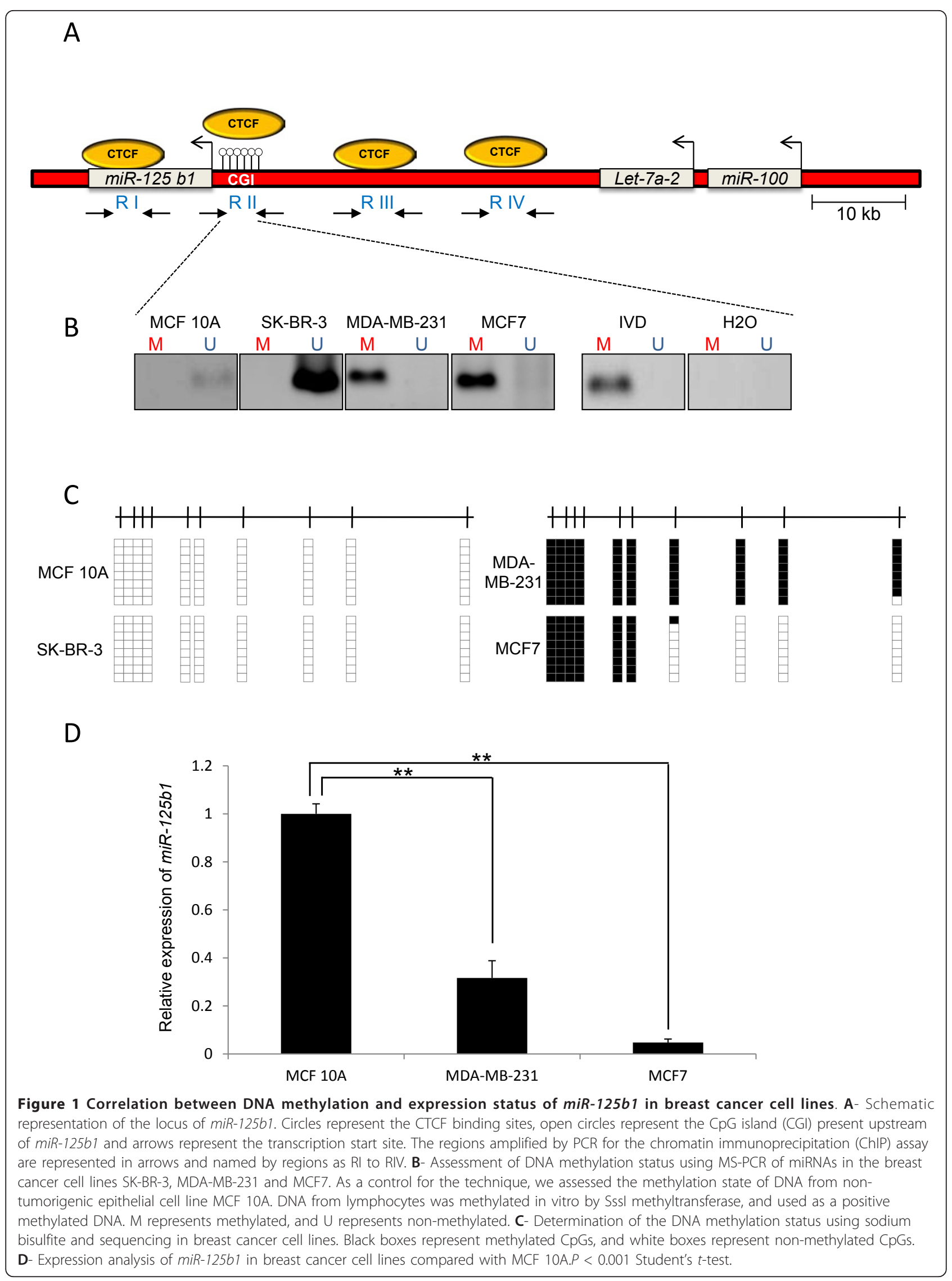




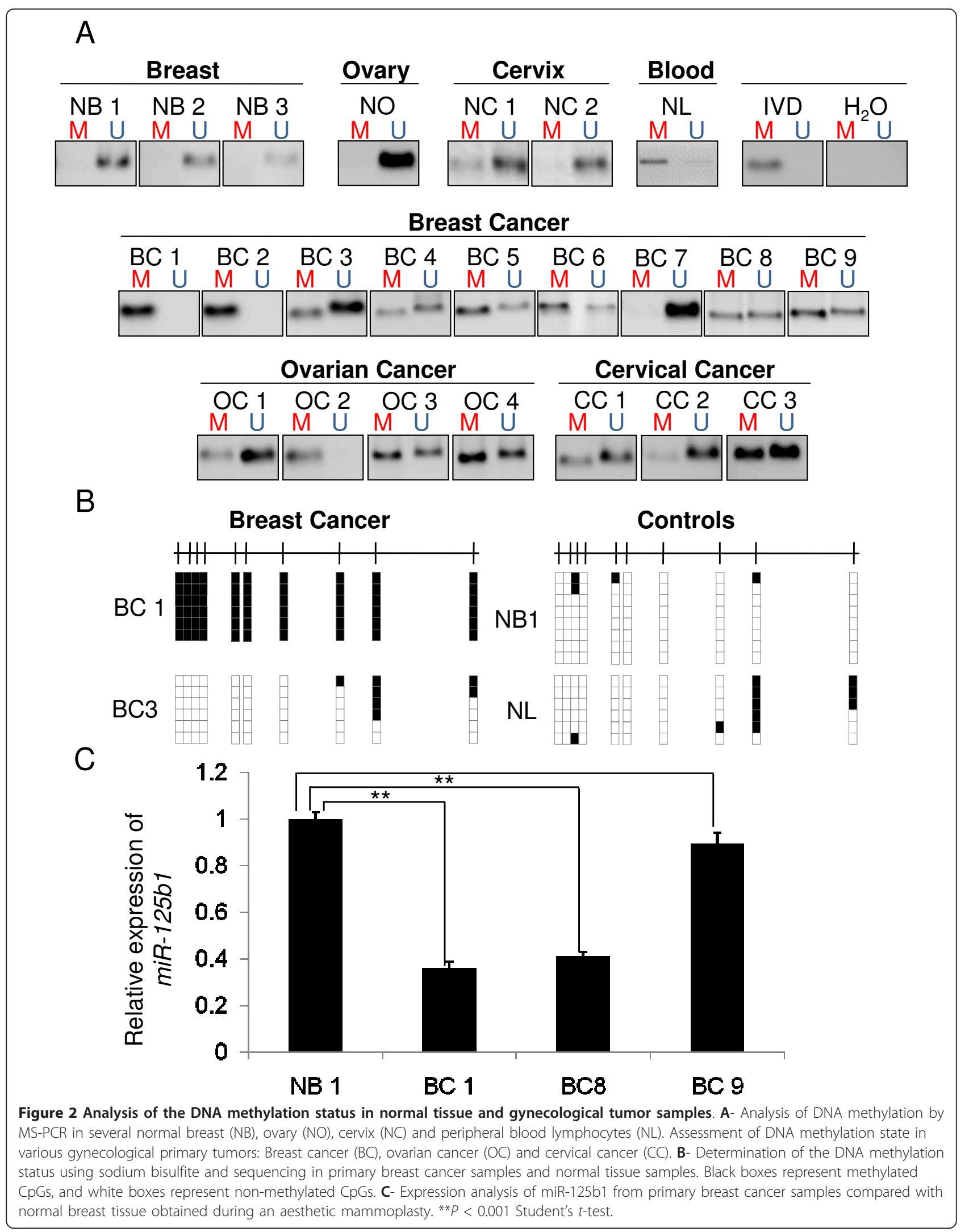


evaluate the presence of this factor using a ChIP assay in cells from normal breast tissue and from two breast cancer samples (Figure 2). This study was extended to four genomic regions of the locus of miR-125b1 (Figure 1A, RI-RIV). In addition, we analyzed the same regions for the presence of several covalent histone modifications such as H3K4me2, a histone mark associated with open chromatin, and the repressive histone marks H3K9me3 and H3K27me3 (Figure 3). An anti-IgG was included as negative control. For this assay, we used four different sets of primers, which amplified three regions located 3' and 5' from the miR-125b1 promoter, where it has been shown that CTCF binds in vivo [21]. In addition, we also used primers corresponding to the promoter region of the gene (Figure 1A). As a positive ChIP assay control for CTCF we evaluated the human p53 gene promoter in all our ChIPs, and for the H3K27me3 histone mark we analyzed the presences of this histone mark at the Myelin transcription factor 1 distal promoter (MYT1) region (Figure 3C).

In normal breast tissue, we observed CTCF enrichment in the four genomic regions, suggesting that this factor is probably linked to the chromatin structure and regulation of the miR-125b1 locus in cells from normal breast tissue (Figure 3A). Subsequently, we obtained chromatin from two breast tumors samples. Using the ChIP assay, we analyzed the covalent histone marks H3K4me2, H3K9me3 and H3K27me3. In the breast tumor cells we observed the loss of CTCF in all regions analyzed (Figure $3 \mathrm{~B})$. The gain of the negative histone mark H3K9me3 was observed in all regions in BC9 and in regions I and IV in $\mathrm{BC} 8$, while $\mathrm{H} 3 \mathrm{~K} 27 \mathrm{me} 3$ was only observed in RI of $\mathrm{BC} 8$ (Figure 3B). These data strongly suggest that the absence of CTCF, together with DNA methylation and some histone repressive marks may contribute to the destabilization of chromatin permissive for transcription and the establishment of an aberrant repressive chromatin configuration of the miR-125b1 locus.

\section{Discussion}

Cancer is primarily a disease that consists of the accumulation of genetic and epigenetic alterations. The epigenetic alterations can lead to the inactivation of tumor suppressor genes and the activation of oncogenes. Because some miRNAs target genes involved in cell cycle control, they have been associated with the functions of tumor suppressor genes and oncogenes [22]. Currently, there is experimental evidence that aberrant patterns of DNA methylation and repressive covalent histone modifications in the promoter regions of miRNAs associated with CpG islands can lead to epigenetic silencing [4]. Some studies have suggested that the loss of DNA methylation in the promoters of some miRNAs (e.g., $m i R-21, m i R-203$ and $m i R-205$ ) in ovarian cancer results in their aberrant overexpression [23]. On the other hand, it has been shown that DNA hypermethylation in the promoters of miR-124a and miR-127 leads to their transcriptional silencing in colon cancer models and influences the expression of two oncogenes, such as BCL6 and CDK6 [4]. The epigenetic silencing of miRNAs has been frequently observed in early stages of breast cancer, including miR-9-1 and the miRNAs of the Let-7 family. Therefore, miRNAs could be good candidates for early tumor markers [24]. Furthermore, some miRNAs have been associated with invasion and metastasis in breast cancer [25]. One of the miRNAs that are deregulated in breast cancer is miR-125b1, and its expression is reduced in this type of cancer, suggesting its importance $[10,26]$.

In the present study, we showed that methylation of DNA in a CpG island upstream of the transcription start site of $m i R-125 \mathrm{~b} 1$ and the gain of repressive histone marks, such as $\mathrm{H} 3 \mathrm{~K} 9 \mathrm{me} 3$ and $\mathrm{H} 3 \mathrm{~K} 27 \mathrm{me} 3$, is a mechanism for the epigenetic silencing of this miRNA. We found that DNA methylation at the $\mathrm{CpG}$ island of $m i R-125 b 1$ is present in the evaluated gynecological tumors. Our results suggest that this methylation negatively affects the miRNA gene expression, indicating that it could be in part responsible for the gene inactivation (Figures 1 and 2).

One of the most studied proteins in vertebrates is the nuclear factor CTCF [27]. This protein has the ability to delimit chromosomal borders, which affect the distinction of transcriptionally active regions from more compact regions in which transcription is repressed. It is currently accepted that CTCF can contribute to the establishment of chromatin domains. This organization promotes the creation of a local chromatin that facilitates communication between regulatory elements, such as promoters or enhancers [28]. Because CTCF has been related with protection against DNA methylation and repressive histone marks propagation, we investigated whether the presence of CTCF is associated with the transcription of miR125b1. Interestingly, in normal breast cells, CTCF is distributed along the locus miR-125b1, whereas in primary breast tumors, we observed the absence of this factor. These data suggest that the absence of CTCF may be related to a gain of aberrant DNA methylation in the CpG island and a gain of repressive histone marks along the four genomic regions studied (Figure 3).

Therefore, we propose that the absence of CTCF along a locus may result in the loss of transcriptionally active borders, and together with DNA methylation and some histone repressive marks, such as H3K27me3, could promote the epigenetic silencing of the miRNA (Figure 4).

Some authors have referred to CTCF as the "glue" that connects the ends of domains, both intra- and 


\section{A Normal Breast}

NB 1

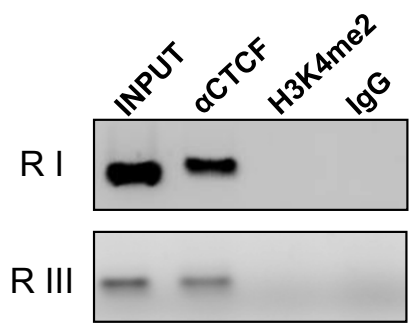

B

\section{Breast Cancer}

BC 8

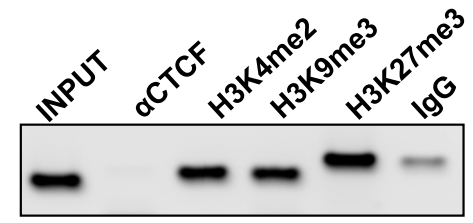

R III

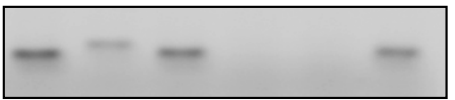

BC 9

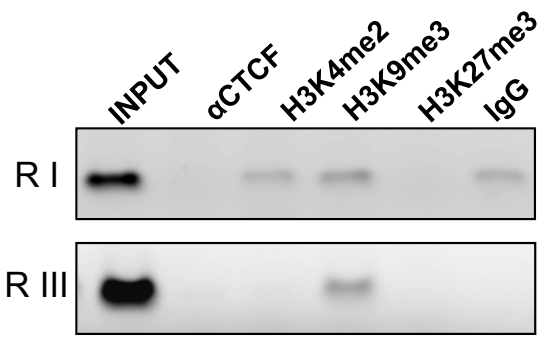

C

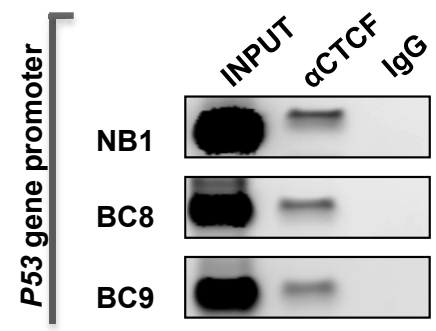

R II

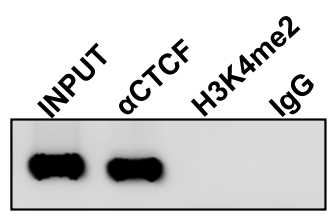

R IV

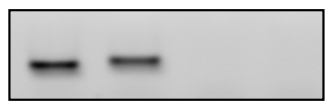

R II

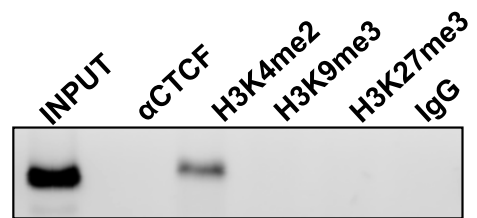

R IV
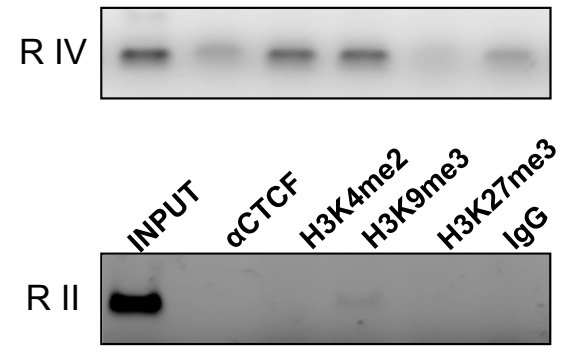

R IV
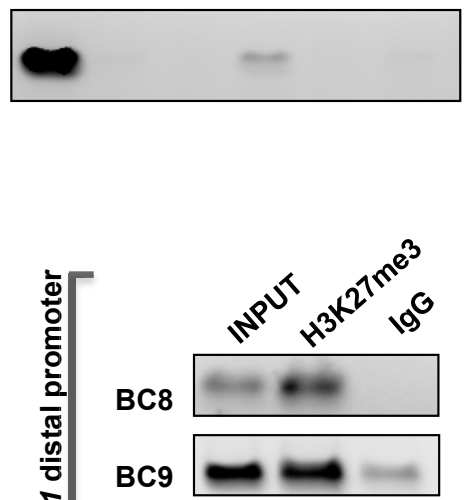

Figure 3 In vivo CTCF binding analysis in miR-125b1 locus and the characterization of covalent histone marks in both normal cells and in primary breast tumors. A- Chromatin immunoprecipitation (ChIP) of CTCF and the histone covalent marks analyzed at the human miR12561 locus in cells obtained from normal breast tissue (NB 1). B- Characterization of CTCF and histone covalent marks in two tumor samples tissue from different patients (BC8 and BC9), input amplification refers as the entire population of DNA. A non-specific lgG antibody was used as a control. C- Positive controls for the ChIP assay. For CTCF we performed a PCR of the p53 gene promoter region in normal breast (NB1) and two tumor samples (BC8 and BC9); for H3K27me3 we evaluated the abundance of the H3K27me3 histone mark at the Myelin transcription factor 1 distal promoter (MYT1) region in two tumor samples (BC8 and BC9). 


\section{Normal Breast

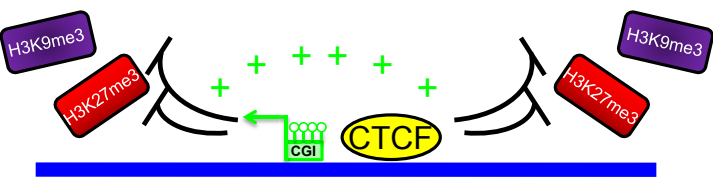 \\ Breast Cancer}

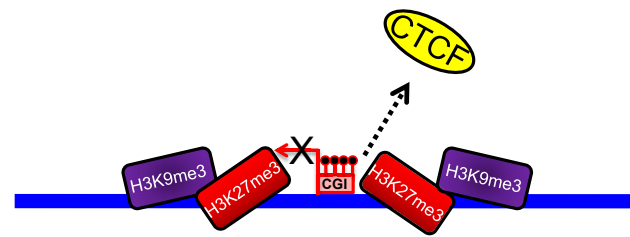

Figure 4 Model of $m i R-125 b 1$ gene silencing, in which the absence of the nuclear factor CTCF is associated with DNA methylation of the CpG island, and the enrichment of repressive histone marks. In normal breast, CTCF might prevent the recruitment of the epigenetic silencing components, such as DNA methylation and repressive histone marks, also favors an open chromatin conformation (green color represent an open chromatin configuration). Meanwhile in breast cancer the loss of CTCF is associated with the CPG island (CGI) methylation and the gain of the repressive histone marks such as $\mathrm{H} 3 \mathrm{~K} 9 \mathrm{me} 3$ and $\mathrm{H} 3 \mathrm{~K} 27 \mathrm{me} 3$.

inter-chromosomally, thus suggesting that deregulation of this factor leads to the aberrant silencing of multiple genes, including miRNAs [29]. These observations are consistent with published data that demonstrate that $m i R-125 b 1$ can be silenced by DNA methylation, which may lead to a worse prognosis in cancer patients [8].

\section{Conclusions}

Our results suggest that a reduction of miR-125b1 expression in cancers, is correlated with methylation, repressive histone marks and loss of CTCF binding at the promoter region.

\section{Acknowledgements \\ This work was supported by the Consejo Nacional de Ciencia y Tecnología (CONACyT: 83959) and Programa de Apoyo a Proyectos de Investigación e Innovación Tecnológica of the Universidad Nacional Autónoma de México (PAPIIT, IN213311). In loving memory of Carlos Hesselbart, "Carlitos".}

\section{Author details}

'Unidad de Investigación Biomédica en Cáncer, Instituto Nacional de Cancerología (INCan)-Instituto de Investigaciones Biomédicas, Universidad Nacional Autónoma de México (UNAM), México, DF, México. ${ }^{2}$ Departamento de Patología, INCan, México, DF, México. ${ }^{3}$ Instituto de Fisiología Celular,
Departamento de Genética Molecular, UNAM, México, DF, México. ${ }^{4}$ Instituto de Investigaciones Biomédicas, UNAM, P.O. Box 70-228, Ciudad Universitaria, 04510 México, DF, México.

\section{Authors' contributions}

ESR and LAH designed methods and experiments, analyzed the results, and drafted the manuscript; RGB, performed the ChIP assays, and drafted the manuscript; FCS carried out the miRNA expression study; RHG and VP performed the histopathology of samples; DC enrolled and followed-up the patients; DP participated in the DNA methylation analysis; CC co-participated in the DNA methylation analysis; FRT analyzed and discussed the results, and drafted the manuscript. All authors have contributed to seen and approved the manuscript.

\section{Competing interests}

The authors declare that they have no competing interests.

Received: 26 September 2011 Accepted: 25 January 2012 Published: 25 January 2012

\section{References}

1. Medina PP, Slack F: MicroRNAs and cancer: an overview. Cell Cycle 2008, 7:2485-2492

2. Melo SA, Esteller M: Dysregulation of microRNAs in cancer: playing with fire. FEBS Lett 2010, 585:2087-2099.

3. Sato F, Tsuchiya S, Meltzer SJ, Shimizu K: MicroRNAs and epigenetics. FEBS 2011, 278:1598-1609.

4. Lujambio A, Ropero S, Ballestar E, Fraga MF, Cerrato C, Setién F, Casado S, Suarez-Gauthier A, Sanchez-Cespedes M, Git A, Spiteri I, Das PP, Caldas C, Miska E, Esteller M: Genetic unmasking of an epigenetically silenced microRNA in human cancer cells. Cancer Res 2007, 67:1424-1429.

5. Laganà A, Russo F, Sismeiro C, Giugno R, Pulvirenti A, Ferro A: Variability in the incidence of miRNAs and genes in fragile sites and the role of repeats and CpG islands in the distribution of genetic material. PLOS One 2010, 5:e11166.

6. Ma L, Weinberg R: MicroRNAs in malignant progression. Cell Cycle 2008, 7:570-572.

7. Visone R, Croce CM: MiRNAs and cancer. Am J Pathol 2009, 174:1131-1138.

8. Zhang Y, Yan LX, Wu QN, Du ZM, Chen J, Liao DZ, Huang MY, Hou JH, Wu QL, Zeng MS, Huang WL, Zeng YX, Shao JY: miR-125b is methylated and functions as a tumor suppressor by regulating the ETS1 protooncogene in human invasive breast cancer. Cancer Res 2011, 71:3552-3562.

9. Feng J, Kim ST, Liu W, Kim JW, Zhang Z, Zhu Y, Berens M, Sun J, Xu J: An integrated analysis of germline and somatic, genetic and epigenetic alterations at 9p21.3 in glioblastoma. Cancer 2012, 118:232-240.

10. Scott GK, Goga A, Bhaumik D, Berger CE, Sullivan CS, Benz CC: Coordinate suppression of ERBB2 and ERBB3 by enforced expression of micro-RNA miR-125a or miR-125b. J BiolChem 2007, 282:1479-1486.

11. Zhou M, Liu Z, Zhao Y, Ding Y, Liu H, Xi Y, Xiong W, Li G, Lu J, Fodstad O, Riker Al, Tan M: MicroRNA-125b confers the resistance of breast cancer cells to paclitaxel through suppression of pro-apoptotic $\mathrm{BCl}-2$ antagonist killer 1 (Bak1) expression. J Biol Chem 2010, 285:21496-21507.

12. Recillas-Targa F, De La Rosa-Velázquez IA, Soto-Reyes E, Benítez-Bribiesca L: Epigenetic boundaries of tumour suppressor gene promoters: the CTCF connection and its role in carcinogenesis. J Cell Mol Med 2006, 10:554-568.

13. Recillas-Targa F, Soto-Reyes E, De la Rosa-Velazquez IA: Insulation of tumor suppressor genes by the nuclear factor CTCF. Biochem Cell Biol 2011, 89:479-488.

14. Butcher DT, Mancini-DiNardo DN, Archer TK, Rodenhiser DI: DNA binding sites for putative methylation boundaries in the unmethylated region of the BRCA1 promoter. Int J Cancer 2004, 111:669-678.

15. Butcher DT, Rodenhiser DI: Epigenetic inactivation of BRCA1 is associated with aberrant expression of CTCF and DNA methyltransferase (DNMT3B) in some sporadic breast tumours. Eur J Cancer 2007, 43:210-219.

16. De La Rosa-Velázquez IA, Rincón-Arano H, Benítez-Bribiesca L, RecillasTarga F: Epigenetic regulation of the human retinoblastoma tumor suppressor gene promoter by CTCF. Cancer Res 2007, 67:2577-2585.

17. Witcher M, Emerson BM: Epigenetic silencing of the p16 (INK4a) tumor suppressor is associated with loss of CTCF binding and a chromatin boundary. Mol Cell 2009, 34:271-284. 
18. Soto-Reyes E, Recillas-Targa F: Epigenetic regulation of the human p53 gene promoter by the CTCF transcription factor in transformed cell lines. Oncogene 2010, 29:2217-2227

19. de Souza Rocha Simonini P, Breiling A, Gupta N, Malekpour M, Youns M, Omranipour R, Malekpour F, Volinia S, Croce CM, Najmabadi H, Diederichs S, Sahin O, Mayer D, Lyko F, Hoheisel JD, Riazalhosseini Y: Epigenetically deregulated microRNA-375 is involved in a positive feedback loop with estrogen receptor alpha in breast cancer cells. Cancer Res 2010, 70:9175-9184.

20. Kirmizis A, Bartley SM, Kuzmichev A, Margueron R, Reinberg D, Green R, Farnham PJ: Silencing of human polycomb target genes is associated with methylation of histone H3 Lys 27. Genes Dev 2004, 18:1592-1605.

21. Barski A, Cuddapah S, Cui K, Roh TY, Schones DE, Wang Z, Wei G, Chepelev I, Zhao K: High-resolution profiling of histone methylations in the human genome. Cell 2007, 129:823-837.

22. Hanahan D, Weinberg RA: Hallmarks of cancer: the next generation. Cell 2011, 144:646-674.

23. Iorio MV, Visone R, Di Leva G, Donati V, Petrocca F, Casalini P, Taccioli C, Volinia S, Liu CG, Alder H, Calin GA, Ménard S, Croce CM: MicroRNA signatures in human ovarian cancer. Cancer Res 2007, 67:8699-8707.

24. Lehmann U, Hasemeier B, Christgen M, Muller M, Romermann D, Langer F, Kreipe $\mathrm{H}$ : Epigenetic inactivation of microRNA gene hsa-mir-9-1 in human breast cancer. J Pathol 2008, 214:17-24.

25. Shi M, Liu D, Duan H, Shen B, Guo N: Metastasis-related miRNAs, active players in breast cancer invasion, and metastasis. Cancer Metastasis Rev 2010, 29:785-799.

26. Tolmachev V: Imaging of HER-2 overexpression in tumors for guiding therapy. Curr Pharm Des 2008, 14:2999-3019.

27. Phillips JE, Corces VG: CTCF: master weaver of the genome. Cell 2009, 26:1194-1211.

28. Handoko L, Xu H, Li G, Ngan CY, Chew E, Schnapp M, Lee CW, Ye C, Ping JL, Mulawadi F, Wong E, Sheng J, Zhang Y, Poh T, Chan CS, Kunarso G, Shahab A, Bourque G, Cacheux-Rataboul V, Sung WK, Ruan Y, Wei CL: CTCF-mediated functional chromatin interactome in pluripotent cells. Nat Genet 2011, 43:630-638.

29. Espinoza CA, Ren B: Mapping higher order structure of chromatin domains. Nat Genet 2011, 43:615-616.

\section{Pre-publication history}

The pre-publication history for this paper can be accessed here: http://www.biomedcentral.com/1471-2407/12/40/prepub

doi:10.1186/1471-2407-12-40

Cite this article as: Soto-Reyes et al:: Disruption of CTCF at the miR$125 \mathrm{~b} 1$ locus in gynecological cancers. BMC Cancer 2012 12:40.

\section{Submit your next manuscript to BioMed Central and take full advantage of:}

- Convenient online submission

- Thorough peer review

- No space constraints or color figure charges

- Immediate publication on acceptance

- Inclusion in PubMed, CAS, Scopus and Google Scholar

- Research which is freely available for redistribution

Submit your manuscript at www.biomedcentral.com/submit
Ciomed Central 